\title{
Division of Entire Functions by Polynomial Ideals
}

\author{
J. Apel \\ Institut für Informatik \\ Universität Leipzig \\ Augustusplatz 10-11 \\ D-04109 Leipzig, Germany \\ apel@informatik.uni-leipzig.de
}

\begin{abstract}
In [ASTW] it was given a Gröbner reduction based division formula for entire functions by polynomial ideals. Here we give degree bounds where the input function can be truncated in order to compute approximations of the coefficients of the power series appearing in the division formula within a given precision. In addition, this method can be applied to the approximation of the value of the remainder function at some point.
\end{abstract}

\section{Introduction}

At almost the same time Hironaka and Buchberger independently developed their theories of standard bases $([\mathrm{Hi}])$ of ideals of formal power series and of Gröbner bases ([Bu]) of polynomial ideals. The theory of graded structures due to Robbiano ([Ro2]) and Mora ([Mo]) unifies both theories and provides a powerful frame for generalizations also to various other rings. The main difference between Gröbner and standard bases consists in the use of noetherian and non-noetherian term orders, respectively. Gröbner like generalizations use noetherian term orders and, therefore, have always terminating reduction relations. Some topological rings allow, and actually require, the use of non-noetherian term orders. Roughly, in such an approach it is important that any infinite reduction sequence converges to some element in the completion of the ring. Examples for rings of such type are rings of power series with the p-adic topology [AMR].

Though, also rings of entire functions are situated somewhere between polynomial rings and rings of formal power series, none of the both generalization directions seems to be applicable to them. Actually, in some sense entire functions inherit the bad properties of both polynomials and formal power series such that neither noetherian nor non-noetherian term orders are applicable. Using a noetherian term order we inherit from the ring of formal power series the non-existence of leading terms for proper power series, i.e. such not being a polynomial. Therefore, we will not have a graded structure. If we assume that the term order is non-noetherian then the reduction process will not be convergent in the topology of local uniform convergence. This we inherit from the subring of polynomial functions. 
In [ASTW] it could be given a partial solution of the problem using a Gröbner like approach, i.e. based on a noetherian term order, which presents a division formula for every where convergent power series by polynomial ideals. This paper will deal only with complex coefficients but large parts of the theory extend also to the real case (see [ASTW]).

The paper is organized as follows: Section 2 will sketch the algebraic and analytic background of the theory. In Section 3 the main ideas developed in [ASTW] concerning the division formula are presented. In Section 4 we show how the entire functions occurring in the division formula can be approximated in an algorithmic way up to a given precision. Furthermore, we show how the value of the reduced power series at given points can be estimated. Finally, we discuss some open problems connected with the theory in Section 5.

The author wants to thank J.Stückrad, P.Tworzewski, and T.Winiarski for the pleasant and fruitful cooperation on the subject of Gröbner theory for everywhere convergent power series.

\section{Preliminaries}

The basic algebraic structures involved in this paper are the polynomial ring $R=\mathbb{C}[X]$, the ring $S=\mathbb{C}[[X]]$ of formal power series, and the ring $E$ of entire functions from $\mathbb{C}^{n}$ into $\mathbb{C}$, i.e. the ring of all formal power series which are convergent at each point of the space $\mathbb{C}^{n}$, in the variables $X=\left(X_{1}, \ldots, X_{n}\right)$ over the field $\mathbb{C}$ of complex numbers. We have the inclusions $R \subset E \subset S$.

It follow some basic ideas of the theories of Gröbner and standard bases. A comprehensive introduction to the Gröbner basis theory can be found in the text book [BW]. For the standard basis case we refer to $[\mathrm{AMR}]$. We assume that the free commutative monoid $T$ generated by $X$, the elements of $T$ will be called terms, is ordered by an admissible term order $\prec$, i.e. by a linear order which is (strong) monotonic with respect to the multiplication. A detailed description and classification of admissible term orders can be found in [Ro1]. Each formal power series $f$ has got a unique representation $f=\sum_{t \in T} c_{t} t, c_{t} \in \mathbb{C}$, in terms of $T$. The set of terms $t$ having non-zero coefficient $c_{t}$ will be called the support $\operatorname{supp}(f)$ of $f$. In case of existence the maximal element of $\operatorname{supp}(f)$ is called the initial term of $f$ with respect to $\prec$ (denotation in $(f)$ ). If $f$ has got an initial term then its coefficient in $f$ will be called the initial coefficient of $f$ with respect to $\prec$. For an arbitrary set $F \subset R$ of polynomials we define the initial $R$-ideal in $(F) \cdot R$ as the ideal generated in $R$ by all initial terms of elements of $F$. The probably most frequently used definition of a Gröbner basis is:

Definition 1. Let $F \subset R$ be a set of non-zero polynomials and $I \subseteq R$ be the ideal generated by $F$. Then $F$ is called a Gröbner basis of $I$ with respect to the admissible term order $\prec$ iff

$$
\operatorname{in}(F) \cdot R=\operatorname{in}(I) \cdot R,
$$

i.e. $F$ and $I$ have the same initial R-ideal. 
Let us assume that $\prec$ is an admissible term order such that each non-zero formal power series has got an initial term. Then the ideal $\operatorname{in}(F) \cdot S$ generated in $S$ by all initial terms of elements of the set $F \subset S$ will be called the initial $S$-ideal of $F$. Similar to Gröbner bases of polynomial ideals we define:

Definition 2. Let $F \subset S$ be a set of non-zero formal power series and $I \subseteq S$ be the ideal generated by $F$. Then $F$ is called a standard basis of $I$ with respect to the admissible term order $\prec$ iff

$$
\operatorname{in}(F) \cdot S=\operatorname{in}(I) \cdot S .
$$

Both, $R$ and $S$, are noetherian rings. Hence, all initial ideals appearing in the Definitions 1 and 2 are finitely generated. Therefore, the existence of a finite Gröbner, respectively standard, basis of the ideal $I$ is ensured. In order to obtain a constructive method for the computation of Gröbner bases we define a division notion:

Definition 3. Let $\prec$ be a noetherian admissible term order and $F=\left\{f_{1}, \ldots, f_{m}\right\}$ be a finite set of non-zero polynomials with initial coefficient 1 . The term $t$ is called reducible modulo $F$ with respect to $\prec$ if $t \in \operatorname{in}(F) \cdot T$. A polynomial is called reducible modulo $F$ with respect to $\prec$ if its support contains at least one term which is reducible modulo $F$ with respect to $\prec$. Otherwise, a polynomial is called irreducible modulo $F$ with respect to $\prec$. Let

$$
\operatorname{in}(F) \cdot T=I_{1} \cup I_{2} \cup \cdots \cup I_{m}
$$

be the disjoint decomposition of the set of all modulo $F$ reducible terms which is defined by $t \in I_{i}$ iff $t$ is reducible modulo $\left\{f_{1}, \ldots, f_{i}\right\}$ but irreducible modulo $\left\{f_{1}, \ldots, f_{i-1}\right\}$ with respect to $\prec(i=1, \ldots, m)$. Let $g \in R$ be a polynomial and $h \in R$ be a polynomial which is irreducible modulo $F$ with respect to $\prec$. Finally, assume that $g$ and $h$ are connected by an equation

$$
g=\sum_{i=1}^{m} h_{i} f_{i}+h
$$

where $t \cdot \operatorname{in}\left(f_{i}\right) \in I_{i}$ for all $i=1, \ldots, m$ and $t \in \operatorname{supp}\left(h_{i}\right)$. Then $h$ is called a normal form of $g$ modulo $F$ with respect to $\prec\left(\right.$ denotation $\left.h=\mathrm{Nf}_{F}(g)\right)$. Equation (1) is called a division formula of $g$ by $F$.

The definition of division in the case of formal power series is analogous. We remark that the polynomials $h$ and $h_{i}(i=1, \ldots, m)$ are uniquely determined by $g, F$ (including the fixed enumeration of the elements $f_{i}$ ) and $\prec$. Hence, fixing $F$ and $\prec$ we get operators mapping from $R$ into $R$ assigning $g$ its normal form $h$ or the cofactor $h_{i}$ of the $i$-th basis polynomial $f_{i}$, respectively. We will call these operators normal form operator or $i$-th cofactor operator, respectively. Note, that all these operators are linear. Furthermore, it is well-known that for Gröbner, respectively standard, bases $F$ the normal form $\mathrm{Nf}_{F}(g)$ depends only on the residue class of $g$ modulo the ideal $I$ generated by $F$. Furthermore, the 
normal forms $\mathrm{Nf}_{F}(g)$ are equal for all Gröbner bases $F$ of $I$ with respect to $\prec$. This justifies to call the normal form modulo a Gröbner, respectively standard, basis $F$ with respect to $\prec$ a normal form modulo the ideal $I$ generated by $F$ with respect to $\prec\left(\right.$ denotation $\mathrm{Nf}_{I}(g)$ ). For the same reason Equation (1) will become a division formula of $g$ by the ideal $I$.

Buchberger's algorithm and appropriate reduction procedures based on Definition 3 provide algorithms for the computation of Gröbner and standard bases. However, there is one serious difference. While it is straight forward to construct a reduction algorithm for the computation of division formulas in the polynomial case an analogue procedure will be non-terminating in the case of formal power series. But in the latter case the reduction sequence will be convergent in the p-adic topology and it can be used the limes of the sequence as the result of the reduction.

At the end of this section we present a convergence criterion which will be used for a similar convergence proof for the reduction process in the case of entire functions with the topology of local uniform convergence. Let $g=\sum_{t \in T} c_{t} t \in S$ and let $r=\left(r_{1}, \ldots, r_{n}\right)$ be an $n$-tuple of positive real numbers. Following [GR] we define the norm

$$
\|g\|_{r}:=\sum_{t \in T}\left|c_{t}\right| t(r),
$$

where $t(r)=r_{1}^{\alpha_{1}} \cdots r_{n}^{\alpha_{n}}$ for $t=X_{1}^{\alpha_{1}} \cdots X_{n}^{\alpha_{n}}$.

Let $B_{r}:=\left\{g \in S \mid \quad\|g\|_{r}<+\infty\right\}$ be the set of formal power series of finite norm. Clearly, we have $E \subseteq B_{r}$ for any $r$. Note, that the topology determined by the system of norms $\|\cdot\|_{r}$ corresponding to all $r$ coincides with the local uniform convergence. The set $B_{r}$ of all formal power series having a finite norm with respect to $r$ forms a Banach algebra. For each subset $\mathcal{D} \subseteq T$ the set $B_{r}(\mathcal{D}):=$ $\left\{g \in B_{r} \mid \operatorname{supp}(g) \subseteq \mathcal{D}\right\}$ of formal power series of $B_{r}$ having a support contained in $\mathcal{D}$ is a closed subspace of $B_{r}$. Hence, $B_{r}(\mathcal{D})$ is a Banach space. Furthermore, the set $E(\mathcal{D})$ of all entire functions having a support contained in $\mathcal{D}$ is a closed subspace of the ring $E$ of entire functions. From this and from elementary facts concerning power series it will follow the convergence criterion:

Lemma 4 [GR]. Let $r_{\nu}=\left(r_{1 \nu}, \ldots, r_{n \nu}\right), \nu=1,2, \ldots$, be a sequence of $n$-tuples of positive real numbers such that

$$
r_{j \nu} \rightarrow+\infty \quad \text { when } \quad \nu \rightarrow \infty, \quad \text { for } j=1,2, \ldots, n \text {. }
$$

If $g_{t} \in E(\mathcal{D})$, for $t \in T$, and

$$
\sum_{t \in T}\left\|g_{t}\right\|_{r_{\nu}}<+\infty, \quad \text { for } \quad \nu=1,2, \ldots
$$

then the series $\sum_{t \in T} g_{t}$ is convergent in $E$ and its sum $g \in E(\mathcal{D})$. 


\section{The division formula}

Our aim is to find a generalization of Gröbner or standard bases to the ring of entire functions. Already the following very simple example shows that there is no hope for the use of a non-noetherian order $\prec$ similar to the case of formal power series. Let $g=X_{1}$ and $F=\left\{1-X_{1}\right\}$. We obtain a reduction sequence $X_{1} \stackrel{F}{\longrightarrow} X_{1}^{2} \stackrel{F}{\longrightarrow} X_{1}^{3} \stackrel{F}{\longrightarrow} \cdots$. Obviously, this sequence is not convergent in the topology of local uniform convergence since, for instance, the values at the point $P=(2)$ tend to infinity. Note, that $1-X_{1}$ is a unit in the ring $S$ of formal power series. Hence, $F \cdot S=S$. But the inclusion $F \cdot E \subset E$ is proper in the case of entire functions.

From now, let $\prec$ be a fixed noetherian admissible term order. Again there is no hope for a complete Gröbner theory since in that case the support of a proper power series will not have a maximal element. Consequently, proper power series will have no initial terms. Furthermore, the ring $E$ is not noetherian in contrary to $R$ and $S$ and, hence, an ideal has no finite Gröbner basis, in general. A first partial solution could be given in [ASTW] where the division formula presented in Definition 3 could be generalized to the case that $g$ is an entire function. It follows the main theorem of that paper:

Theorem 5 [ASTW]. Let $\prec$ be a noetherian admissible term order,

$$
g=\sum_{t \in T} c_{t} t \in E
$$

be an entire function, and $I \subset R$ be an ideal generated by the finite set $F=$ $\left\{f_{1}, \ldots, f_{m}\right\}$ of non-zero polynomials with initial coefficient 1 with respect to $\prec$. Then there exists a division formula

$$
g=\sum_{j=1}^{m} h_{j} f_{j}+h
$$

where $h \in E$ and $h_{j} \in E$ for $j=1, \ldots, m$. Furthermore, the support of $h$ contains only terms which are irreducible modulo $F$ with respect to $\prec$. If $F$ is a Gröbner basis of $I$ then $h$ is the only entire function which is congruent to $g$ modulo IE and which is irreducible modulo $F$ with respect to $\prec$.

Proof. We will sketch only the basic ideas. The aim is to show that normal form operator and cofactor operators can be linearly extended from $R$ to $E$. So, the central problem is to check that the linear extensions are sound. We ask whether the infinite sum

$$
\sum_{t \in T} c_{t} \mathrm{Nf}_{F}(t)
$$

exists. Note, that the coefficients of each term in the sum are infinite sums of complex numbers. So, we need at least the convergence of all these sums in order to have a well-defined object. 
Let $\mathcal{D}$ be the set of all terms which are irreducible modulo $F$ with respect to $\prec$. Then there exist a sequence $r_{\nu}=\left(r_{1 \nu}, \ldots, r_{n \nu}\right), \nu=1,2, \ldots$, of $n$-tuples of positive real numbers, satisfying

$$
r_{j \nu} \rightarrow+\infty \text { when } \quad \nu \rightarrow \infty, \quad \text { for } j=1,2, \ldots, n,
$$

such that the following conditions are satisfied for all $\nu=1,2, \ldots$ and all polynomials $\hat{g} \in R$ :

i) $\left\|\mathrm{Nf}_{F}(\hat{g})\right\|_{r_{\nu}}+\sum_{i=1}^{m}\left\|\hat{h}_{i}\right\|_{r_{\nu}} \leq\|\hat{g}\|_{r_{\nu}}$,

ii) $\left\|\mathrm{Nf}_{F}(\hat{g})\right\|_{r_{\nu}} \leq\|\hat{g}\|_{r_{\nu}}$,

iii) $\left\|\hat{h}_{i}\right\|_{r_{\nu}} \leq\|\hat{g}\|_{r_{\nu}}$ for all $1 \leq i \leq m$,

where

$$
\hat{g}=\sum_{i=1}^{m} \hat{h}_{i} f_{i}+\mathrm{Nf}_{F}(\hat{g})
$$

is the division formula of $\hat{g}$ by $F$ defined in Definition 3. In particular, condition ii) holds for terms $t$, hence, $\left\|\mathrm{Nf}_{F}(t)\right\|_{r_{\nu}} \leq\|t\|_{r_{\nu}}$.

Since $g \in B_{r_{\nu}}$ we have $\|g\|_{r_{\nu}}<+\infty$. Consequently,

$$
\sum_{t \in T}\left\|c_{t} \mathrm{Nf}_{F}(t)\right\|_{r_{\nu}} \leq \sum_{t \in T}\left|c_{t}\right|\|t\|_{r_{\nu}}<+\infty .
$$

Applying Lemma 4 it will follow that the sum exists and that it is not only a formal power series but an entire function which in addition has a support containing only terms of $\mathcal{D}$.

Using condition iii) it can be proved in a similar manner that the linear extension of the $i$-th cofactor operator to $E$ is sound.

In $[\mathrm{ASTW}]$ it was also shown that the normal form operator is continuous. Using this and linearity the uniqueness of the normal form modulo a Gröbner basis follows in the usual way. Again, the uniqueness justifies the denotation $\mathrm{Nf}_{I}(g)$ in the Gröbner basis case.

\section{Approximations}

In the preceding section we presented a constructive method for the division of entire functions by polynomial ideals. We have to compute a Gröbner basis of the polynomial ideal and then to reduce the entire function modulo this Gröbner basis. But, in general, the reduction process will not stop and we are faced with the problem of approximation. We put the following questions:

- Where we can truncate the entire function $g$ such that the coefficients of $\mathrm{Nf}_{I}(g)$ and $h_{j}$ differ from those obtained by reduction of the truncated power series only within some given precision $\epsilon$ ?

- How to compute an approximation of the value of $\mathrm{Nf}_{I}(g)$ at some given point $P \in \mathbb{C}^{n}$ ? 
Let $g \in E$ be an entire function and $F=\left\{f_{1}, \ldots, f_{m}\right\}$ be the reduced Gröbner basis, i.e. the minimal autoreduced Gröbner basis containing only polynomials with initial coefficient 1 , of the (non-trivial) polynomial ideal $I \subset R$ with respect to the noetherian admissible term order $\prec$. We set $\omega:=\max _{c \in \operatorname{coef}(F)}|c|$, where coe $f(F)$ is the set of all coefficients of polynomials contained in $F$. Furthermore, let $\lambda$ denote the maximal length, i.e. number of terms, of polynomials from $F$. Finally, we define $\kappa:=\omega(\lambda-1)$ as measure of the complexity of $F$ in case that $I$ is not a monomial ideal. For monomial ideals $I$, i.e. $\lambda=1$, we set $\kappa:=1$. In the sequel we will estimate the coefficients of normal form and cofactors of the division formula of $g$ by $F$ in terms of the constant $\kappa$.

Let $w=\left(w_{1}, \ldots, w_{n}\right)$ be an $n$-tuple of positive natural numbers. We will call $w$ a positive weight vector and define the $w$-degree of a term $t=X_{1}^{\alpha_{1}} \cdots X_{n}^{\alpha_{n}}$ by $w \operatorname{deg}(t)=\sum_{i=1}^{n} w_{i} \alpha_{i}$. Bayer proved in his thesis [Ba] that for any polynomial ideal $I$ and any admissible term order $\prec$ there exists a positive weight vector $w$ such that $F \subset R$ is Gröbner bases of $I$ with respect to $\prec$ if and only if $F$ is Gröbner basis of $I$ with respect to the admissible term order $\prec_{w}$ comparing first the $w$-degrees and breaking ties by $\prec$. We can choose $w$ such that, in addition, for each element of $F$ the $w$-degree of the initial term is strictly greater than the $w$-degree of any other term contained in its support. The division formulas of $g$ by $I$ will be the same for both orders for each $g \in E$. So, without loss of generality, we can assume that the order $\prec$ itself has the above properties of $\prec_{w}$. In the following we fix a suitable weight vector $w$. Under this assumption we can enumerate the terms of $T$ by $t_{1}, t_{2}, \ldots$ such that $t_{i} \prec t_{i+1}$ for all $i=1,2, \ldots$ We start our investigation in the elementary case of the division formula of a term $t_{i}$ by $F$ :

$$
t_{i}=\sum_{j=1}^{m} h_{i, j} f_{j}+\operatorname{Nf}_{I}\left(t_{i}\right) .
$$

The complex number coefficients appearing in the entire functions contained in Equation (2) are denoted according to the equations:

$$
\begin{aligned}
\mathrm{Nf}_{I}\left(t_{i}\right) & =\sum_{l=1}^{\infty} b_{i, l} t_{l} \quad \text { and } \\
h_{i, j} & =\sum_{l=1}^{\infty} a_{i, j, l} t_{l} \quad(1 \leq j \leq m) .
\end{aligned}
$$

A first trivial observation is $b_{i, l}=a_{i, j, l}=0$ for $l>i$.

Approximation of normal form coefficients The coefficients of the normal form of a term can be estimated by:

Lemma 6. Let $b_{i, l}$ be the coefficients of the normal form of the $i$-th term $t_{i}$ modulo $F$ with respect to $\prec$ from Equation (3). Then we have the bound:

$$
\left|b_{i, l}\right| \leq \kappa^{w d e g\left(t_{i}\right)} \text { for all } l=1,2, \ldots .
$$


Proof. We proceed by induction on the $w$-degree of $t_{i}$. First of all we observe that the assertion is satisfied for any term $t_{i}$ which is irreducible modulo $F$ with respect to $\prec$ since in this case we have $b_{i, i}=1$ and $b_{i, l}=0$ for $l \neq i$. We assumed $I$ to be a proper ideal. Hence, $t_{1}=1$ is irreducible modulo $F$ and Inequality (5) holds for the only term of $w$-degree 0 .

Assume now, $t_{i}$ is reducible modulo $F$ with respect to $\prec$ and $w \operatorname{deg}\left(t_{i}\right)>1$. Consider the division formula

$$
t_{i}=\sum_{j=1}^{m} h_{i, j} f_{j}+\mathrm{Nf}_{F}\left(t_{i}\right)
$$

of $t_{i}$ by $F$. Definition 3 implies that there is exactly one pair $1 \leq k \leq m$ and $t \in T$ such that $t \in \operatorname{supp}\left(h_{i, k}\right)$ and $t \cdot \operatorname{in}\left(f_{k}\right)=t_{i}$. Furthermore, it holds $h_{i, k}=t+\hat{h}_{i, k}$, where $\hat{h}_{i, k}=0$ or in $\left(\hat{h}_{i, k}\right) \prec t$. We have

$$
t_{i}-t f_{k}=\sum_{j=1}^{p} d_{j} t_{j},
$$

where $d_{p} \neq 0$ and $p<i$. By the assumption that the $w$-degree of the initial term of the element $f_{k} \in F$ is strictly greater than the $w$-degree of any other term of $\operatorname{supp}\left(f_{k}\right)$ it follows

$$
w \operatorname{deg}\left(t_{j}\right)<w \operatorname{deg}\left(t_{i}\right) \text { for all } j \leq p .
$$

So, by induction assumption the Inequality (5) is satisfied for each coefficient $b_{j, l}$ of $\mathrm{Nf}_{F}\left(t_{j}\right)(j=1, \ldots, p)$. Obviously,

$$
\left|d_{j}\right| \leq \omega
$$

and

$$
\#\left\{d_{j} \mid d_{j} \neq 0\right\}<\lambda,
$$

i.e. the number of non-zero coefficients $d_{j}$ is less than $\lambda$. Linearity of the normal form operator implies

$$
\mathrm{Nf}_{F}\left(t_{i}\right)=\mathrm{Nf}_{F}\left(t_{i}\right)-\mathrm{Nf}_{F}\left(t f_{k}\right)=\sum_{j=1}^{p} d_{j} \mathrm{Nf}_{F}\left(t_{j}\right)
$$

Hence, we have the equation

$$
b_{i, l}=\sum_{j=1}^{p} d_{j} b_{j, l} .
$$

Finally, using the Inequalities (6), (7), (8) and Equation (9) we obtain the estimation

$$
\left|b_{i, l}\right| \leq(\lambda-1) \cdot \omega \cdot \kappa^{w \operatorname{deg}\left(t_{i}\right)-1}=\kappa^{w \operatorname{deg}\left(t_{i}\right)} .
$$


Let $\mathrm{Nf}_{I}(g)=\sum_{j=1}^{\infty} d_{j} t_{j}$ be the normal form of the entire function $g=$ $\sum_{i=1}^{\infty} c_{i} t_{i}$ modulo $I$. The sum

$$
\mathrm{Nf}_{I}(g)=\sum_{i=1}^{\infty} c_{i} \mathrm{Nf}_{F}\left(t_{i}\right)=\sum_{i=1}^{\infty} c_{i} \sum_{l=1}^{\infty} b_{i, l} t_{l}
$$

is independent of the order of summation, hence,

$$
\mathrm{Nf}_{I}(g)=\sum_{l=1}^{\infty}\left(\sum_{i=1}^{\infty} c_{i} b_{i, l}\right) t_{l} .
$$

Comparison of coefficients yields

$$
d_{j}=\sum_{i=1}^{\infty} c_{i} b_{i, j}
$$

Using estimation (5) it will follow

$$
\left|d_{j}\right| \leq \sum_{i=1}^{\infty}\left|c_{i}\right| \kappa^{w \operatorname{deg}\left(t_{i}\right)}=\|g\|_{r_{w}},
$$

where $r_{w}=\left(\kappa^{w_{1}}, \ldots, \kappa^{w_{n}}\right)$.

Since $g$ is an entire function the above norm is finite and we have an upper bound for the coefficient size. The following theorem shows that the problem of finding approximations of the coefficients of $\mathrm{Nf}_{I}(g)$ within a given precision can be reduced to the ability of norm computations.

Theorem 7. Let $g \in E$ with

$$
\mathrm{Nf}_{I}(g)=\sum_{j=1}^{\infty} d_{j} t_{j}
$$

and $\epsilon$ be a positive real number. Furthermore, let $g=h+h^{\prime}$ be a decomposition of $g$ such that $h \in R$ is a polynomial having the normal form

$$
\mathrm{Nf}_{I}(h)=\sum_{j=1}^{\infty} a_{j} t_{j}
$$

and $h^{\prime} \in E$ is an entire function satisfying $\left\|h^{\prime}\right\|_{r_{w}}<\epsilon$ with respect to the norm vector $r_{w}$ defined above. Then

$$
\left|d_{j}-a_{j}\right|<\epsilon \text { for all } j=1,2, \ldots .
$$

Proof. The assertion follows immediately from (10) and linearity of $\mathrm{Nf}_{I}$. 
It follow some remarks on the truncation (with respect to $\prec_{w}$ ) of $g$ such that sufficiently good approximations of the coefficients of $\mathrm{Nf}_{F}(g)$ can be read off already from the normal form of the polynomial obtained by truncation of $g$.

Given the reduced Gröbner basis $F$ the computations of $w, \kappa$, and $r_{w}$ are algorithmic. The bottleneck will be the computation of $N=\|g\|_{r_{w}}$ within a given precision $\frac{\epsilon}{2}$. Assume we were able to do this. Then there is no essential difficulty finding the term $t_{l}$ such that $\left|N-\left\|\sum_{i=1}^{l} c_{i} t_{i}\right\|_{r_{w}}\right|<\epsilon$. This implies $\left\|\sum_{i=l+1}^{\infty} c_{i} t_{i}\right\|_{r_{w}}<\epsilon$ since our norm is additive for power series with disjoint supports. Hence, $h=\sum_{i=1}^{l} c_{i} t_{i}$ and $h^{\prime}=\sum_{i=l+1}^{\infty} c_{i} t_{i}$ satisfy the assumptions of Theorem 7 and reduction of the polynomial $h$ provides the coefficients of $\operatorname{Nf}_{F}(g)$ within the wished precision.

Approximation of cofactor coefficients Now, we will show that the same truncation is suitable also for a sufficiently good approximation of the coefficients of the cofactors $h_{j}$ appearing in the division formula of $g$ modulo $F$. We start with an estimation of the coefficients $a_{i, j, l}$ of Equation (4).

Lemma 8. Let $t_{i}$ be the $i$-th term with respect to $\prec$. Then the coefficients $a_{i, j, l}$ of Equation (4) satisfy the condition

$$
\left|a_{i, j, l}\right| \leq \kappa^{\text {wdeg }\left(t_{i}\right)} \text { for all } i, l=1,2, \ldots \text { and } j=1, \ldots, m .
$$

Proof. The proof is similar to that of Lemma 6. But instead of the linearity of the normal form operator we have to use the linearity of the cofactor operators.

By linearity of the $j$-th cofactor operator we have

$$
h_{j}=\sum_{i=1}^{\infty} c_{i} h_{i, j}
$$

for the entire function $g=\sum_{i=1}^{\infty} c_{i} t_{i}$, the $j$-th cofactor $h_{j}$ of the division formula of $g$ by $F$, and the cofactors $h_{i, j}$ from Equation (2). Similar preparations as for Inequality (5) yield $|d| \leq\|g\|_{r_{w}}$ for any coefficient $d$ occurring in $h_{j}$.

Theorem 9. Let $g \in E$ with division formula

$$
g=\sum_{i=1}^{m} h_{i} f_{i}+\mathrm{Nf}_{I}(g)
$$

and $\epsilon$ be a positive real number. Furthermore, let $g=h+h^{\prime}$ be a decomposition of $g$ such that $h \in R$ is a polynomial with division formula

$$
h=\sum_{i=1}^{m} \hat{h}_{i} f_{i}+\mathrm{Nf}_{I}(h)
$$


modulo $F$ and $h^{\prime} \in E$ is an entire function satisfying $\left\|h^{\prime}\right\|_{r_{w}}<\epsilon$ with respect to the norm vector $r_{w}$. Then

$$
\left|\operatorname{coe} f\left(h_{i}, t_{j}\right)-\operatorname{coe} f\left(\hat{h}_{i}, t_{j}\right)\right|<\epsilon \text { for all } i=1, \ldots, m \text { and } t_{j} \in T,
$$

where coef $(f, t)$ denotes the coefficient of the term $t$ in the entire function $f$.

Proof. The proof is analogue to that of Theorem 7 .

Approximation of values at given points Since we are working with functions $g=\sum_{i=1}^{\infty} c_{i} t_{i}$ it is natural to ask for their value $g(P)$ at a given point $P \in \mathbb{C}^{n}$. For the zero point $O=(0, \ldots, 0)$ we can immediately give a positive answer, namely $g(O)=c_{1}$. For an arbitrary point $P$ we can transform the problem to the value computation at zero by expanding $g$ at the point $P$. The expansion of $g$ at $P=\left(P_{1}, \ldots, P_{n}\right)$ can be obtained by application of the automorphism $\iota$ of $E$ defined by $X_{i} \mapsto X_{i}-P_{i}$ to $g$. Clearly, $g(P)=\iota(g)(O)$. However, the computation of $\iota(g)$ is not algorithmic, too, and again we need truncations for the approximation.

We introduce a set $Y=\left(Y_{1}, \ldots, Y_{n}\right)$ of new variables and define the polynomial rings $R_{Y}=\mathbb{C}[Y], R_{X, Y}=R[Y]$, and the corresponding rings $E_{Y}$ and $E_{X, Y}$ of entire functions. Furthermore, we set $T_{Y}$ and $T_{X, Y}$ the sets of terms in $Y$ and $X \cup Y$, respectively. Let $T_{Y}$ be ordered by $\prec_{Y}$ in the same way as $T$, i.e. $Y^{\alpha} \prec_{Y} Y^{\beta}$ iff $X^{\alpha} \prec X^{\beta}$. In $T_{X, Y}$ we use an admissible term order which coincides with $\prec$ and $\prec_{Y}$ on $T$ and $T_{Y}$, respectively, and which is such that any term of $T_{Y}$ is smaller than any term containing a variable of $X$. Clearly, $E, E_{Y} \subset E_{X, Y} . E$ and $E_{Y}$ are isomorphic by the natural isomorphism $X_{i} \mapsto Y_{i}$ $(1 \leq i \leq n)$. The composition of $\iota$ and this isomorphism provides an isomorphism $\varphi: E \rightarrow E_{Y}$ mapping $X_{i}$ to $Y_{i}-P_{i}$. We have

$$
g(P)=\varphi(g)(O) \text { for any } g \in E .
$$

Let $J \subset R_{X, Y}$ be the ideal generated by $G=\left\{X_{i}-Y_{i}+P_{i} \mid i=1,2, \ldots, n\right\}$. It is easy to verify that $G$ is reduced Gröbner basis with respect to $\prec_{X, Y}$ and that the computation of the image $\varphi(g)$ can be performed by reduction of $g \in E$ modulo $G$, i.e

$$
\mathrm{Nf}_{J}(g)=\varphi(g) \text { for all elements } g \in E .
$$

Lemma 10. Let $I \subset R$ be a polynomial ideal and $F=\left\{f_{1}, \ldots, f_{m}\right\} \subset I$ be a Gröbner basis of $I$ with respect to $\prec$. Then $\varphi(F)$ is Gröbner basis of the polynomial ideal $\varphi(I) \subset R_{Y}$ with respect to $\prec_{Y}$, where $\varphi, R_{Y}$ and $\prec_{Y}$ have the same meaning as introduced above.

Proof. Let $0 \neq g \in \varphi(I)$. Since $F$ is Gröbner basis we have

$$
\varphi^{-1}(g)=\sum_{i=1}^{m} h_{i} f_{i},
$$


where $h_{i}=0$ or in $\left(h_{i} f_{i}\right) \preceq \operatorname{in}\left(\varphi^{-1}(g)\right)$ for $1 \leq i \leq m$. Applying the isomorphism $\varphi$ we obtain a representation

$$
g=\sum_{i=1}^{m} \varphi\left(h_{i}\right) \varphi\left(f_{i}\right)
$$

of $g$ in terms of $\varphi(F)$. The particular structure of the isomorphism $\varphi$ and the choice of the term order $\prec_{Y}$ ensure that in $(f)=X^{\alpha}$ implies $\operatorname{in}(\varphi(f))=Y^{\alpha}$ for arbitrary non-zero polynomials $f \in R$. Therefore, $\varphi\left(h_{i}\right)=0$ or in $\left(\varphi\left(h_{i} f_{i}\right)\right)=$ $\operatorname{in}\left(\varphi\left(h_{i}\right) \varphi\left(f_{i}\right)\right) \preceq \operatorname{in}(g)$ for $1 \leq i \leq m$. Hence,

$$
\operatorname{in}(\varphi(I)) \cdot R_{Y} \subseteq \operatorname{in}(\varphi(F)) \cdot R_{Y} .
$$

This and trivial facts show that $\varphi(F)$ satisfies the conditions of Definition 1.

Lemma 11. Let $I \subset R$ be a polynomial ideal and $F \subset I$ a Gröbner basis of $I$ with respect to $\prec$. Furthermore, let $G, J, R_{X, Y}, \varphi$, and $\prec_{X, Y}$ be as above. Then the set $G \cup \varphi(F)$ is a Gröbner basis of $J+I \cdot R_{X, Y}$ with respect to $\prec_{X, Y}$.

Proof. $\varphi(F)$ is Gröbner basis of $\varphi(I)$ with respect to $\prec_{Y}$ according to Lemma 10. Since the initial terms of the elements of $G$ depend only on $X$ and those of the elements of $\varphi(F)$ only on $Y$, the union of the Gröbner bases is Gröbner basis of the ideal sum with respect to $\prec_{X, Y}$.

After these preparations we return to our initial problem, which was to calculate an approximation of the value of the entire function $\mathrm{Nf}_{I}(g)$ at the point $P$. According to the Equations (12) and (13) this is equivalent to the approximation of the coefficient of the term $t_{1}=1$ in $\varphi\left(\mathrm{Nf}_{I}(g)\right)=\mathrm{Nf}_{J}\left(\mathrm{Nf}_{I}(g)\right)$. A direct attempt to use the results about the approximation of the normal form coefficients involves one difficulty, namely, we would have to approximate the coefficients of $\mathrm{Nf}_{I}(g)$ first and then to continue the calculation with the approximated data. In order to do this we would need some knowledge about the norm error of our approximation of $\mathrm{Nf}_{I}(g)$. But the estimation of the norm error will remain an open problem of this paper. However, the following theorem shows that $\mathrm{Nf}_{J}\left(\mathrm{Nf}_{I}(g)\right)$ can be calculated using a single normal form calculation modulo only one Gröbner basis.

Theorem 12. Let $g \in E$ be an entire function, $I \subset R$ be a polynomial ideal and $F=\left\{f_{1}, \ldots, f_{m}\right\} \subset I$ a Gröbner basis of $I$ with respect to the admissible term order $\prec$. Furthermore, let $R_{Y}, R_{X, Y}, E_{Y}, E_{X, Y}, \prec_{Y}$, and $\prec_{X, Y}$ be as introduced above. Finally, let $P=\left(P_{1}, \ldots, P_{n}\right) \in \mathbb{C}^{n}$ be a point, $J \subset R_{X, Y}$ the ideal generated by the reduced Gröbner basis $G=\left\{X_{i}-Y_{i}+P_{i} \mid i=1,2, \ldots, n\right\}$, and $\varphi: E \rightarrow E_{Y}$ the isomorphism defined by $X_{i} \mapsto Y_{i}-P_{i}$. Then

$$
\varphi\left(\mathrm{Nf}_{I}(g)\right)=\mathrm{Nf}_{J}\left(\mathrm{Nf}_{I}(g)\right)=\mathrm{Nf}_{J+I R_{X, Y}}(g) .
$$


Proof. Clearly, $\mathrm{Nf}_{J}\left(\mathrm{Nf}_{I}(g)\right) \equiv g \bmod J+I R_{X, Y}$ and $\operatorname{supp}\left(\mathrm{Nf}_{J}\left(\mathrm{Nf}_{I}(g)\right)\right) \subseteq T_{Y}$. Let $Y^{\alpha} \in \operatorname{supp}\left(\mathrm{Nf}_{J}\left(\mathrm{Nf}_{I}(g)\right)\right)$. There must exists a term $t \in \operatorname{supp}\left(\mathrm{Nf}_{I}(g)\right)$ which is multiple of $X^{\alpha}$ due to the particular structure of $G$. Consequently, $Y^{\alpha}$ is irreducible modulo $\varphi(F)$ and $\mathrm{Nf}_{J}\left(\mathrm{Nf}_{I}(g)\right)$ is irreducible modulo $G \cup \varphi(F)$. In conclusion, $\mathrm{Nf}_{J}\left(\mathrm{Nf}_{I}(g)\right)$ is the unique normal form of $g$ modulo $J+I R_{X, Y}$ with respect to $\prec_{X, Y}$.

In summary we can compute the value of $\mathrm{Nf}_{I}(g)$ at the point $P$ by

\section{Corollary 13.}

$$
\mathrm{Nf}_{I}(g)(P)=\mathrm{Nf}_{J+I R_{X, Y}}(g)(O)
$$

The approximation of the right hand side can be solved by estimating the absolute term of the normal form of $g$ modulo $G \cup\left\{\mathrm{Nf}_{J}\left(f_{1}\right), \ldots, \mathrm{Nf}_{J}\left(f_{k}\right)\right\}$ with respect to $\prec_{X, Y}$.

Note, that the feature expressed in Theorem 12 that the normal form calculation modulo an ideal sum may be replaced by sequential normal form calculations modulo the summands is very exceptional in the theory of Gröbner bases and strongly depends on the simple structure of $J$ and the use of suitable term orders. In our particular situation we have a second nice feature which is far from being self-evident, namely, the very easy computation of the Gröbner basis of $J+I \cdot R_{X, Y}$ which requires only reduction of the elements of one basis modulo the other.

\section{Closing remark}

We presented a constructive method for the division of entire functions by polynomial ideals. The main problem of our method is the norm estimation of entire functions. The difficulty of this task depends on the concrete power series under consideration. The first question which had to be answered is which type of representation should be assumed for the input power series.

Another question which we could not solve in this paper is the computation of an approximation of the normal form such that the error of its norm is within some given precision.

The coefficient bounds given in this paper are independent on the term to which they belong. There are some obvious possible improvements of the bounds depending on the concrete term under consideration. In this sense we could ask also for the error of coefficients of "large" enough terms. This could be a way to attack the problem of computing approximations such that the norm error is within some range.

A very important question is whether there are criterions which allow to prove that a coefficient is exactly zero. In particular, this is important for the question whether the normal form of some entire function is a polynomial. Unfortunately, up to now we are not able to give any hint how to solve the problem, unless $I$ is a monomial ideal. 
A last remark concerns the input basis of the ideal. Looking for approximations one could ask whether also approximations of the input data are sufficient. It is well known, that a Gröbner basis computation starting from polynomials with only approximated coefficients makes no sense. Of course, we cannot expect a continuous behaviour since little changes of the coefficients of the input polynomials may change the generated ideal drastical, e.g. the ideal dimension may change. So we conclude, that in principal we have to make the hard assumption that the polynomial ideal has to be given exactly. The only errors allowed, i.e. which will effect a continuous behaviour, are little errors at the coefficients of the polynomials of the reduced Gröbner basis $F$ leaving the initial ideal unchanged.

\section{References}

[AMR] Alonso, M.E., Mora, T., Raimondo, M.: A computational method for algebraic power series. J. Pure Appl. Algebra 77 (1992) 1-38.

[ASTW] Apel, J., Stückrad, J., Tworzewski, P., Winiarski, T.: Reduction of everywhere convergent power series with respect to Gröbner bases. J. Pure Appl. Algebra, (to appear).

[Ba] Bayer, D.: The division algorithm and the Hilbert Scheme. Ph.D. Thesis, Harvard University (1982).

[BW] Becker, T., Weispfenning, V.: Gröbner Bases, A Computational Approach to Commutative Algebra. Springer, New York, Berlin, Heidelberg (1993).

[Bu] Buchberger, B.: Ein Algorithmus zum Auffinden der Basiselemente des Restklassenringes nach einem nulldimensionalen Polynomideal. Ph.D. Thesis, Univ. Innsbruck (1965).

[GR] Grauert, H., Remmert, R.: Analytische Stellenalgebren. Springer (1971).

[Hi] Hironaka, H.: Resolution of singularities of an algebraic variety over a field of characteristic zero. Annals of Math. 79 (1964) 109-326.

[Mo] Mora, T.: Seven variations on standard bases. Preprint, Univ. di Genova, Dip. di Mathematica, N. 45 (1988).

[Ro1] Robbiano, L.: Term orderings on the polynomial ring. L.N.C.S. 204 (1985) $513-517$.

[Ro2] Robbiano, L.: On the theory of graded structures. J.Symb.Comp. 2 (1986) 139-170.

This article was processed using the $\mathrm{AT}_{\mathrm{E}} \mathrm{X}$ macro package with LLNCS style 